\title{
Cervical Spinal Cord Injury Leads to Injury and Metabolic Dysfunction of the Lung
}

\author{
Running title: SCI leads to ALI
}

Emily E. Huffman ${ }^{1,2}$, Brittany E. Dong ${ }^{3}$, Harrison A. Clarke ${ }^{1}$, Lyndsay E.A. Young ${ }^{4,5}$, Derek
B. Allison ${ }^{4,6}$, Ramon C. Sun ${ }^{1,2}$, Christopher, M. Waters ${ }^{3,7}$, Warren, J. Alilain* ${ }^{1,2}$

Corresponding author: Warren J. Alilain, Ph.D.

Associate Professor

B469 BBSRB

741 S. Limestone Avenue

Lexington, KY 40536

859-257-3219

warren.alilain@uky.edu

\section{Author Contribution Statement}

EEH and WJA conceived of and designed the work for this project. EEH acquired, analyzed, and interpreted its data, drafted, and revised this manuscript. BED and CMW were integral in the interpretation of data and training for this work. DBA provided assessment of the lung pathology. HAC, LEAY, and RCS provided the metabolomic work and analysis for this project. All authors assisted in editing this manuscript.

Keywords: spinal cord injury, lung injury, acute respiratory distress syndrome

Impact statement: This research establishes a model of cervical spinal cord injury-induced lung injury that can be used to study the progression of lung injury after SCI. This approach demonstrates the physiological consequences of SCI that contribute to acute lung injury.

\section{Funding Information:}

This publication was financially supported by NIH R01 NS101105 (WJA). NIH R01

HL131526 (CMW), NIH R01 HL151419 (CMW), NIH R01 AG066653 (RCS), and 1R21NS121966-01 (WJA).

This publication was supported by the National Center for Research Resources and the National Center for Advancing Translational Sciences, National Institutes of Health, through Grant UL1TR001998. The content is solely the responsibility of the authors and does not necessarily represent the official views of the NIH.

Research reported in this publication was supported by an Institutional Development Award (IDeA) from the National Institute of General Medical Sciences of the National Institutes of Health under grant number P20GM103527.

\footnotetext{
${ }^{1}$ Department of Neuroscience, University of Kentucky College of Medicine, Lexington, KY, United States.

${ }^{2}$ Spinal Cord and Brain Injury Research Center, University of Kentucky College of Medicine, Lexington, KY, United States.

${ }^{3}$ Department of Physiology, University of Kentucky College of Medicine, Lexington, KY, United States.

${ }^{4}$ Markey Cancer Center, University of Kentucky, Lexington, KY, United States.

${ }^{5}$ Department of Molecular and Cellular Biochemistry, University of Kentucky, Lexington, KY, United States.

${ }^{6}$ Department of Pathology and Laboratory Medicine, University of Kentucky College of Medicine, Lexington, KY, United States.

${ }^{7}$ Saha Cardiovascular Research Center, University of Kentucky College of Medicine, Lexington, KY, United States.
} 


\section{Acknowledgements}

We thank Jessica Newton, M.S., Chris Calulot, B.S., Daimen Britch, B.S., and Lydia

Strattan, Ph.D. for their assistance in completing experiments. We also thank Lydia Strattan,

Ph.D., Jessica Newton, M.S., and Aaron Silverstein, B.S., B.S., for their assistance revising this manuscript. 


\begin{abstract}
High-cervical spinal cord injury (SCI) often disrupts respiratory motor pathways and disables breathing in the affected population. Moreover, cervically injured individuals are at risk for developing acute lung injury (ALI), which predicts substantial mortality rates. While the correlation between ALI and SCI has been found in the clinical setting, the field lacks an animal model to interrogate the fundamental biology of this relationship. To begin to address this gap, we performed an experimental cervical SCI and assessed lung injury in adult rats. We demonstrate that animals display signs of ALI two weeks post-SCI. We also observed aberrant $\mathrm{N}$-glycan metabolism determined by matrix-assisted laser desorption/ionization mass spectrometry imaging. Collectively, we establish for the first time a model of ALI after SCI at an acute time point that can be used to monitor the progression of lung damage, as well as identify potential targets to ameliorate ALI.
\end{abstract}




\section{Introduction}

Cervical spinal cord injuries (SCIs) can silence descending signals from the brainstem that control diaphragmatic activity. This injury can consequently impair breathing, making individuals prone to the development of profound respiratory complication ${ }^{1-3}$. In fact, respiratory complication is the leading cause of morbidity and mortality for cervical SCI individuals ${ }^{4}$. Two common forms of these complications found in SCI are acute lung injury (ALI) and its most severe form, acute respiratory distress syndrome (ARDS). Both complications introduce significant risks of mortality that may occur in association with a myriad of etiologies such as trauma, pneumonia, shock, aspiration, and sepsis ${ }^{5,6}$. Inflammatory responses to these neutrophil mediated injuries contribute damage to the vascular endothelium and alveolar epithelium, where long-term repercussions can persist even after resolution of lung injury ${ }^{7,8}$.

Recent evidence suggests that persons with an SCI hold a significant risk of developing ARDS/ALI. Most notably, those with a cervical injury hold the greatest risk and the highest mortality rate compared to other levels of SCI ${ }^{9}$. This observation is not limited to SCI alone. In fact, ARDS/ALI has been found in humans following neurotrauma, including vertebral column fracture, stroke, and traumatic brain injury ${ }^{9-11}$. In addition to the implications for lung damage and inhibition of ventilatory function, there is evidence that ARDS/ALI also impacts neurologic outcomes following central nervous system injury ${ }^{12}$. These substantial consequences present an exigent call to characterize the peripheral repercussions of SCI. While many comorbidities and complications of SCI have gained attention in recent years, the effect of a cervical SCI on the lungs, especially the characterization of their health, function, and cellular composition, has not been explored in the animal model ${ }^{13,14}$. Currently, the field lacks an animal model to interrogate 
the molecular underpinning of ALI after cervical SCI, which is a major barrier to identifying therapeutic windows and treatment opportunities.

In this study, we report a model of cervical SCI-induced ALI/ARDS in rats. We found classic markers of lung injury after SCI that persist two weeks after the initial time of cervical injury. We performed a thorough assessment of lung injury including severity of edema, protein infiltration in the bronchoalveolar lavage fluid, neutrophil and cellular levels, cytokine concentrations, $\mathrm{N}$-linked glycans, and histopathological reports which enabled a thorough assessment of lung injury. This study demonstrates that features of lung injury ensue after experimental cervical SCI, establishing a promising model of ALI/ARDS at an acute time point that can be used to characterize and monitor the progression of lung injury after SCI, as well as provide a means to test potential therapies.

\section{Materials and Methods}

\section{Animals}

All procedures were in accordance with Institutional Animal Care and Use Committee (IACUC) guidelines. Thirty female Sprague Dawley retired breeder rats (12 months) from Charles River Laboratories, Inc. were housed under standard light/dark cycles with ad libitum access to food and water. Animals were monitored daily following surgery.

\section{C2 Hemisection Spinal Cord Injury}

C2 Hemisection was conducted as previously described ${ }^{15}$. Briefly, animals were anaesthetized with $4 \%$ isoflurane and prepared for surgery by shaving and cleaning from the ears to the shoulders with alternating bouts of Betadine and $70 \%$ ethanol. Body temperature was maintained throughout the procedure by a heating pad underneath the animal. Under aseptic conditions, a dorsal midline incision was performed to expose the cervical region of the spinal 
column. A C2 laminectomy allowed for visualization of the dorsal cervical spinal cord. After durotomy, a 27-gauge needle was bent and inserted into midline at C2 and dragged laterally to sever the left hemi-cord. This process was repeated for a total of three times to ensure completion. After $\mathrm{C} 2$ hemisection $(\mathrm{C} 2 \mathrm{Hx})$, the layers of musculature were sutured together and the skin was clipped closed. The animals were given Buprenorphine $(0.02-0.05 \mathrm{mg} / \mathrm{kg})$, Carprofen $(5 \mathrm{mg} / \mathrm{kg})$, and saline when removed from anesthesia. These post-operative care procedures were continued for three days following injury.

\section{Experimental Design}

Fifteen rats were given a cervical SCI, while another fifteen were left intact as naïve. Sham animals were not included as the injury mimics a vertebral column fracture, which has also been shown to induce ALI/ARDS in the clinical setting, rendering it an ineffective control for studying the effects of an SCI on the lungs ${ }^{9}$. The SCI and naïve animals were each randomly assigned to three groups: the edema group, BAL group, and histology/MALDI-MSI group. These 3 groups each contained 5 naïve animals and 5 SCI animals. This division allows for each group to have 10 animals total, with 20 lungs to study.

\section{Pre-collection Procedure and Sacrifice}

Animals were deeply anesthetized under $4 \%$ isoflurane (SomnoSuite Low-Flow Anesthesia System) and subsequently overdosed by isoflurane administration 2 weeks after SCI. Rats received a laparotomy and the abdominal aorta was transected as a secondary means of sacrifice. All procedures were conducted immediately post-mortem.

\section{Bronchoalveolar Lavage Fluid Collection}

Following sacrifice, the ribcage was reflected and suture was guided under each primary bronchus, preparing for ligation. An 18-gauge tracheal cannula connected to a $3 \mathrm{~mL}$ syringe 
filled with $2 \mathrm{~mL}$ of sterile, cold $0.1 \%$ EDTA was inserted without force into the left bronchus and ligated with suture. The syringe was compressed slowly, taking 1 minute to fill the lung. Insertion was verified by inflation of the lung. After a 1-minute pause, bronchoalveolar lavage (BAL) fluid was withdrawn slowly, taking 1 minute to remove from the left lung, and deposited into sterile vials on ice. This was repeated by attaching a second syringe filled with $2 \mathrm{~mL}$ of sterile solution to the inserted cannula. Keeping the cannula in the respiratory tract, the needle was then guided into the right primary bronchus and the procedure was repeated. In total, $4 \mathrm{~mL}$ of lavaged fluid were collected from each lung.

\section{Pulmonary Edema}

Following sacrifice, the heart and lungs were pulled out of the animal en bloc, cutting all connective tissue and vessels in the process. Once removed, the lungs were separated, blotted until visibly dry, and placed in pre-weighed $15 \mathrm{~mL}$ conical vials. The wet lungs were weighed before placement in a hybridization oven at 65 degrees Celsius uncapped. After 48 hours, the desiccated lungs were weighed again. The wet/dry weight ratio was calculated by dividing the mass of the wet lung by the mass of the dry lung.

\section{Histology.}

A 16-gauge blunted cannula was inserted into the trachea and secured with suture. Sixty mL cold PBS were pushed through the right ventricle of the heart to clear the lungs of blood. The cannulated lungs were then removed from the pleural cavity along with the heart and pressure fixed $\left(25 \mathrm{~cm} \mathrm{H}_{2} \mathrm{O}\right)$ en bloc with $10 \%$ formalin. The left and right lungs were then dissected from each other and placed in tissue cassettes in $10 \%$ formalin for 24 hours. Cassettes were transferred to $70 \%$ ethanol for another 24 hours. Lungs were promptly embedded in paraffin, serially 
sectioned at $5 \mu \mathrm{m}$, and mounted on slides. Injury severity was assessed by a blinded pathologist after hematoxylin and eosin staining (H\&E stain).

Cytokine Assessment

Collected BAL was centrifuged at $200 \mathrm{x}$ g for 10 minutes at $4{ }^{\circ} \mathrm{C}$ and the supernatant was immediately frozen. TNF- $\alpha$, IL-1 $\beta$, IL-4, IL-6, and keratinocyte chemoattractant/ human growthrelated oncogene $(\mathrm{KC} / \mathrm{GRO})$ concentrations were measured in the supernatant via mesoscale discovery assay (MSD), which uses electrochemiluminescence as a detection technique for higher sensitivity.

\section{Protein Assessment}

Protein concentrations were measured via Bicinchoninic Acid assay (BCA). Briefly, 25 $\mu \mathrm{L}$ of BAL supernatant were mixed with $100 \mu \mathrm{L}$ of reagent and incubated at $37^{\circ} \mathrm{C}$ for 30 minutes. Protein concentration was analyzed at $562 \mathrm{~nm}$ and compared to a standard curve constructed using known protein concentrations.

\section{Cellular Assessments}

Pellets from centrifuged BAL were resuspended in $500 \mu \mathrm{L}$ PBS. Cell counts were performed with Trypan blue exclusion and approximately 30,000 cells were cytospun onto microscopy slides. After drying, the slides were fixed and stained with a Diff-Quik stain. Total neutrophils were measured by morphologically identifying neutrophils per 200 cells observed.

\section{Matrix-Assisted Laser Desorption Ionization Mass Spectrometry Imaging}

Formalin-fixed paraffin embedded lungs (FFPE) were processed as previously described ${ }^{16}$. In brief, FFPE lungs were sectioned at $5 \mu \mathrm{m}$ on glass slides. Tissues were dewaxed and rehydrated followed by an antigen retrieval process in citraconic anhydride buffer. Recombinant PNGase F $(0.1 \mu \mathrm{g} / \mathrm{mL})$ was applied by a M5 TM robotic Sprayer (HTX Technologies LLC, 
Chapel Hill, NC). Following incubation in a humidity chamber for 2 hours, slides were desiccated for 24 hours. The following day, $7 \mathrm{mg} / \mathrm{mL}$ of alpha-Cyano-4-hydroxycinnamic acid in $50 \%$ acetonitrile with $0.1 \%$ TFA were applied to each slide by the robotic sprayer. Slides were subsequently stored in a desiccator prior to MALDI-MSI analysis.

A Waters Synapt G2Si mass spectrometer (Waters Corporation, Millford, MA) equipped with a Nd:YAG UV laser with a spot size of $100 \mu \mathrm{m}$ was used to detect $\mathrm{N}$-glycans at X and Y coordinates of $100 \mu \mathrm{m}$. Following data acquisition, mass spectra were analyzed by High Definition Imaging (HDI) Software (Waters Corporation) for mass range 500-3500m/z. Three regions of interest were determined for each lung, pixel intensities were averaged and normalized by total ion current. Representative N-glycans were generated by Glycworkbench.

\section{Statistical Analysis}

All data are presented as the mean \pm SEM. All numerical data were analyzed by a twotailed unpaired student t-test. For all analyses, $\mathrm{P}<0.05$ was considered significant. Following MALDI-MSI analysis of N-gylcans, Metaboanalyst 5.0 was used to generate heatmaps and principal component analyses ${ }^{17}$.

\section{Results}

\section{Pulmonary Edema Developed after SCI}

To determine whether there was an excess of fluid in the lungs after acute SCI, we evaluated the wet/dry ratio from the lungs of naïve and 14dpi SCI animals. The wet/dry ratio has been found to correlate with the results of other measures of lung injury ${ }^{18}$. We found that $\mathrm{C} 2 \mathrm{Hx}$ elicited a significant increase in the wet/dry ratio of the lungs of SCI animals $(4.89 \pm 0.06)$ compared to naïve lungs $(4.72 \pm 0.04)(\mathrm{P}<0.05)($ Fig. 1A). There was a trend towards a greater 
wet/dry ratio in the left lungs compared to the right lungs following $\mathrm{C} 2 \mathrm{Hx}(\mathrm{N}=5)$ (Figure 1B).

However, with a small sample size, we were unable to evaluate the significance of this trend ${ }^{19}$.

SCI Increases BAL Cell Counts but Not Protein Levels in Rats

To further characterize the severity of injury, we evaluated the migration of cells into the lungs after SCI ${ }^{20}$. Following SCI, the total number of cells increased significantly $(1.41 \pm 0.04$ $\left.\mathrm{x} 10^{7}\right)$ compared to naïve lungs $\left(4.31 \pm 0.02 \times 10^{6}\right)(\mathrm{P}<0.05)$. Additionally, a differential cell count revealed that the number of neutrophils was elevated in SCI animals $\left(1.10 \pm 0.18 \times 10^{5}\right)$ compared to naïve $\left(7.79 \pm 1.29 \times 10^{3}\right)(\mathrm{P}<0.0001)($ Fig 2 A-D). Using these observations, we estimated that neutrophils comprised $1.8 \%$ of the BAL prior to injury and $8.6 \%$ post-SCI. These results show that not only did the number of cells increase in the BAL, but the ratio of neutrophils found in the lungs also increased after SCI. However, as shown in Fig. 2 E-F, protein levels in the BAL were not significantly increased in the SCI rats $(0.133 \pm 0.006 \mu \mathrm{g} / \mu \mathrm{l})$ compared to naïve $(0.124 \pm 0.005 \mu \mathrm{g} / \mu \mathrm{l})(\mathrm{Fig} .2 \mathrm{E}-\mathrm{F})$. Again, there appeared to be a trend towards the left lung having both a greater cell count and a larger neutrophil count compared to the right lung $(\mathrm{N}=5)$.

\section{SCI Modulate Cytokine Levels in BAL}

To further investigate the increase in cells found in the BAL after SCI, we measured levels of the neutrophil chemoattractant $\mathrm{KC} / \mathrm{GRO}$, as well as other indicators of inflammation including the cytokines TNF- $\alpha$, IL-1 $\beta$, and IL-6, whose concentrations have been observed to increase after lung injury ${ }^{21-23}$. MSD results showed significantly increased levels of KC/GRO $(100.0 \pm 12.33 \mu \mathrm{g} / \mu \mathrm{l})$ and TNF- $\alpha(0.50 \pm 0.06 \mu \mathrm{g} / \mu \mathrm{l})$ in SCI animals compared to naïve $(64.04 \pm 2.28 \mu \mathrm{g} / \mu \mathrm{l}),(0.31 \pm 0.03 \mu \mathrm{g} / \mu \mathrm{l})$ respectively (Fig. $3 \mathrm{~A}-\mathrm{D}) . \mathrm{IL}-1 \beta$ levels remained unchanged two weeks after injury (3.06 \pm 0.41$)$ compared to naïve $(2.23 \pm 0.25 \mu \mathrm{g} / \mu \mathrm{l})$ (Fig E-F). 
Levels of IL- 6 were under the range of detection in 15 out of 20 samples. However, the in-range samples belonged to SCI animals.

\section{No Histopathologic Changes Were Observed in the Lungs After Acute SCI}

Histological analysis by a blinded pathologist using H\&E staining revealed similar findings in both SCI and naïve animals. More specifically, both groups had rare scattered small foci containing foamy pulmonary alveolar macrophages, mild chronic interstitial inflammation, and mild interstitial fibrosis. However, the majority of the examined lung parenchyma was relatively normal in appearance in all cases. With a more severe acute lung injury, we would expect to see an accumulation of intra-alveolar immune infiltrates, alongside more robust and diffuse interstitial inflammatory infiltrate and microvascular congestion. Lack of histopathology features suggest we are at early stages of lung injury, thereby establishing the treatment window for the future testing of therapeutic options.

\section{SCI Is Associated With Changes in N-linked Glycosylation of the Lung}

In order to determine whether SCI caused metabolic changes in lung tissue, we performed crude carbohydrate analysis of the lung using periodic acid-schiff (PAS) stain that revealed broad changes in complex carbohydrates ${ }^{24-26}$. Similar to H\&E analysis, PAS staining showed no detectable changes between naïve and ALI following SCI (Fig. 5C and 6C). To further investigate whether N-linked glycan changes occur in ALI, we performed in situ analysis of N-linked glycomics by enzyme-assisted matrix-assisted laser desorption/ionization (MALDI) mass spectrometry imaging. First, we performed multiple multivariate analysis that include supervised clustering heatmap and partial least squares discriminant analysis (PLS-DA) on both left and right lung with ALI (Fig. 5A-B \& 6A-B). Both clustering heatmap and PLS-DA analysis uniquely separated naïve and SCI injured lungs (Fig. 5A-B \& 6A-B). Interestingly, 
variable importance in projection (VIP) values from PLS-DA reveal heterogenous changes in Nlink glycans between left and right lungs (Fig. 5B \& 6B). Finally, we performed gross tissue structural analysis using the MALDI dataset and found that changes in glycans are consistent across entire lung regions and not confined to specific regions (Fig. 5D \& 6D). Collectively, MALDI imaging analysis of N-linked glycomics suggest distinct aberrant N-glycan metabolism is associated with ALI after SCI and these changes are lobe dependent. Further, spatial analysis showing universal changes across entire lobe suggests the possibility of mechanical defects after SCI rather than localized cellular damage.

\section{Discussion}

A majority of individuals with SCI develop secondary complications within the first two weeks of injury ${ }^{27}$. Specifically, individuals with cervical SCI have a high incidence of respiratory complications and can develop ARDS/ALI after injury ${ }^{9,28}$. We investigated this clinical observation in the rat model in an effort to capture the effects of SCI on the lungs. Overall, this study demonstrates that mild lung injury does occur following cervical SCI. We observed signs of mild ARDS/ALI at two weeks after cervical SCI with trends towards greater injury in the lung ipsilateral to SCI. These findings establish a model of ALI/ARDS at an acute time point that can be used to characterize and monitor the progression of lung injury after SCI, as well as a means to investigate potential therapeutic strategies.

ARDS/ALI is a progressive disease with various forms of damage occurring at different timepoints depending upon the initiating event. The temporal developments of lung injury following SCI have not been established in the rat model ${ }^{29}$. However, our data appear to indicate the exudative stage of ALI, with evidence of edema and infiltration of cells into the alveoli 
without indications of fibrosis. These observations two weeks after SCI suggest a disruption of the epithelial-endothelial barrier in the alveoli.

ARDS/ALI characteristically results in the increased permeability of the alveolarcapillary barrier. The damage of this barrier facilitates the influx of fluid into the alveoli. We assessed edema by calculating the wet/dry ratio for each lung and we found that animals with SCI had increased edematous fluid in both the left and right lungs compared to uninjured controls. Interestingly, despite utilizing a unilateral SCI model, there was no significant difference in edema between the left and the right lung, suggesting that our model of SCI did not specifically target either lung. However, there is a reasonably strong trend that the ipsilateral lung had a greater wet/dry ratio compared to the contralateral lung in SCI animals. With a small sample size, we were unable to evaluate the significance of this trend ${ }^{19}$. Another indicator of lung injury is the recruitment of immune cells into the airspace of the lungs. As expected, we observed primarily alveolar macrophages in the cell counts of uninjured animals. After SCI, the population of cells in the alveoli increased primarily due to the recruitment of neutrophils.

Because we observed a substantial increase in both total cell number and neutrophils in the BAL after SCI, we evaluated $\mathrm{KC} / \mathrm{GRO}$ as it is known to mediate neutrophil recruitment and activation ${ }^{30}$. We found an increase in $\mathrm{KC} / \mathrm{GRO}$ in the BAL following SCI. We demonstrated that the increase in neutrophils and $\mathrm{KC} / \mathrm{GRO}$ persisted for 14 days following SCI. KC/GRO has functional homology with IL-8 with chemotactic and proinflammatory activity in the rodent ${ }^{31}$. High levels of $\mathrm{KC} / \mathrm{GRO}$ in the BAL from ARDS/ALI patients are associated with increased neutrophils in the injured lungs ${ }^{32}$.

Knowing that $\mathrm{KC} / \mathrm{GRO}$ levels were elevated, we assayed cytokines involved in its inflammatory cascade ${ }^{33}$. We observed increased levels of TNF- $\alpha$ in the collected BAL fluid 
from SCI rats. TNF- $\alpha$ has been proposed as a mediator of lung injury and its neutralization ameliorates the injury ${ }^{21,22}$. Interestingly, there were no significant changes in IL-1 $\beta$ levels after SCI and IL-6 levels were undetectable in naïve and SCI animals. The inability to detect changes in IL-1 $\beta$ and IL-6 may be due to these cytokines' varying temporal profiles, with IL-6 peaking hours after induction of lung injury in the mouse model, for example ${ }^{17}$. However, more timepoints need to be evaluated after SCI to provide further insights into the progression of lung injury following SCI.

Increased protein concentration in the BAL is another indicator of lung injury and vascular permeability in both rodents and humans ${ }^{34}$. Damage to the alveolar-capillary barrier allows protein from the blood to enter the lungs at the level of the capillaries ${ }^{35}$. However, despite increased wet/dry ratios in SCI rats, we did not find substantial increases in protein concentration in the BAL after SCI. One potential explanation for this finding is that fluid accumulation may have occurred due to increased hydrostatic pressure rather than loss of the alveolar-capillary barrier function. However, we do not have direct evidence for this since we did not measure pulmonary vascular pressures in this model. Our injury model is one of an indirect induction of lung injury, consistent with other forms of trauma ${ }^{36}$. With the paralysis of the left hemidiaphragm as opposed to the entire diaphragm, the injury is not as severe as a complete transection or contusion SCI.

After 14 days, SCI rats showed no histopathologic differences compared to the control rats. The vast majority of the examined lungs were histologically unremarkable with both groups showing rare foci of non-specific findings. However, the presence of these limited findings in both the SCI rats and controls suggests that they are unrelated to the induced SCI. Additionally, the lack of prominent indications of diffuse alveolar damage is consistent with the lack of 
increased protein in the BAL. These results were expected in the acute setting of SCI, as there has likely not yet been a long enough time-course to result in significant pathologic findings. The changes that we found in Figures 1-3 may manifest into histopathological damage in more chronic timepoints following SCI.

Although we did not observe significant evidence of histological lung injury, we investigated changes in $\mathrm{N}$-linked glycan metabolism as a potential early indicator of the development of lung injury. N-linked glycan biosynthesis is an understudied facet of glucose metabolism. In the lung, N-linked glycans are crucial for the differentiation of bronchoalveolar stem and alveolar type 2 cells to form the alveolar and bronchiolar lining ${ }^{37-39}$. Further, N-linked glycans are critical components of mucins and surfactant proteins that maintain the liquid-air interface, reduce surface tension, and provide lubrication for the mechanical action of the lung ${ }^{40,41}$. Collectively, MALDI imaging analysis of N-linked glycomics suggests distinct aberrant Nglycan metabolism is associated with ALI after SCI and that these changes are lobe dependent. Further, spatial analysis shows universal changes across the entire left and right lobes. These findings support the possibility of widespread changes in metabolism after SCI, that could potentially impact lung progenitor cell differentiation, oxygen/CO2 exchange, and movement of the lung. With these important implications in mind, aberrant $\mathrm{N}$-linked glycan phenotype in our ALI model warrant further investigation.

ALI/ARDS is often induced in animal models with a direct inflammatory insult to the lungs ${ }^{42}$. However, ALI/ARDS can also manifest as a result of indirect insults such as sepsis, burns, and trauma via inflammatory mechanisms ${ }^{36,43}$. After SCI, there is an intense systemic inflammatory response that affects whole organ systems ${ }^{44,45}$. Lung damage has been found in rodent models after thoracic-level SCIs with evidence for systemic inflammation as the cause 
${ }^{13,46}$. However, lung injury after cervical SCI cannot be solely attributed to inflammation.

Individuals with cervical SCIs are at higher risk for the development of ARDS/ALI and have more severe ARDS/ALI than at any other injury level ${ }^{9}$. This severity is likely because an injury to the cervical level of the spinal cord affects breathing function while simultaneously generating a general systemic inflammatory response ${ }^{47}$. Our results suggest this explanation may be the case, as we demonstrate that the lung ipsilateral to the SCI trends towards more severe signs of ARDS/ALI. However, the significance of these differences was immeasurable due to small sample size ${ }^{19}$. This observation may be a result of the left $\mathrm{C} 2 \mathrm{Hx}$ injury model used in this study. In this model, the innervating signal to the left hemidiaphragm is severed above the level of the phrenic motor nucleus. This injury results in paralysis of the left hemidiaphragm, leaving the operation of the right hemidiaphragm intact. The desuetude of the left hemidiaphragm affects the pressure-differential in the left pleural cavity and lowers the tidal volume of injured animals ${ }^{15}$. With a reduced capacity of the lungs to perform gas-exchange, ALI/ARDS can ensue ${ }^{48}$. Future experiments will be directed toward elucidating the side-specific differences in the lungs following unilateral SCI.

We provide a means to investigate potential therapeutic strategies to ameliorate the onset and progression of lung injury after SCI in the rat model. Based on our collective findings, it would be pertinent to look to the aberrant metabolic profile of the lungs following SCI. Specifically, advances are being made in glycan-based immunotherapeutics in the treatment of breast and lung cancer ${ }^{49}$. A broader use of these therapeutics may one day be applied to lung injury after SCI to target the changes in metabolism we observe in the lungs following SCI.

In summary, our studies demonstrated that animals with a cervical SCI display signs of mild ALI/ARDS including, but not limited to marked increases in alveolar neutrophil counts, 
heightened levels of proinflammatory cytokines and neutrophil-activating KC/GRO, intraalveolar edema, and changes in $\mathrm{N}$-glycan metabolism 14 days after injury. Collectively, this study establishes an SCI-induced ALI model that can be developed further and used to identify potential targets to ameliorate respiratory distress and lung injury after SCI.

\section{Author Disclosure Statement}

No competing interests of any kind, financial or otherwise, exist for this manuscript. 
A

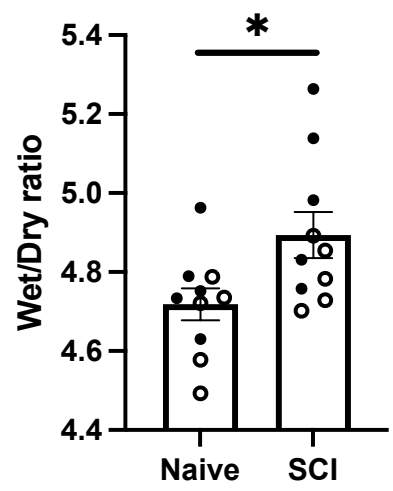

B

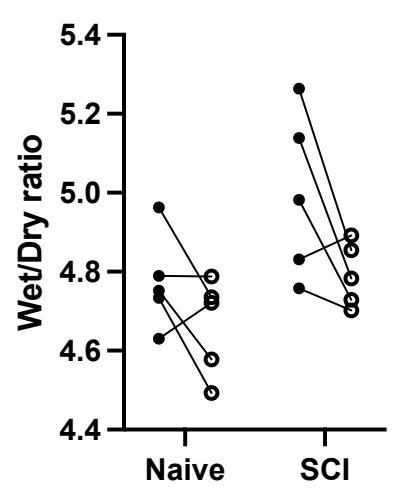

$\rightarrow$ L Lung

$\rightarrow$ R Lung

Figure 1.

Wet/Dry ratios of excised lungs were significantly increased two weeks post-SCI compared with naïve rats $(A ; N=10, P<0.05)$. The left lung presented a trend towards a greater wet/dry ratio $(B$; $\mathrm{N}=5$ ). The significance of this finding was not analyzed due to small Ns. 

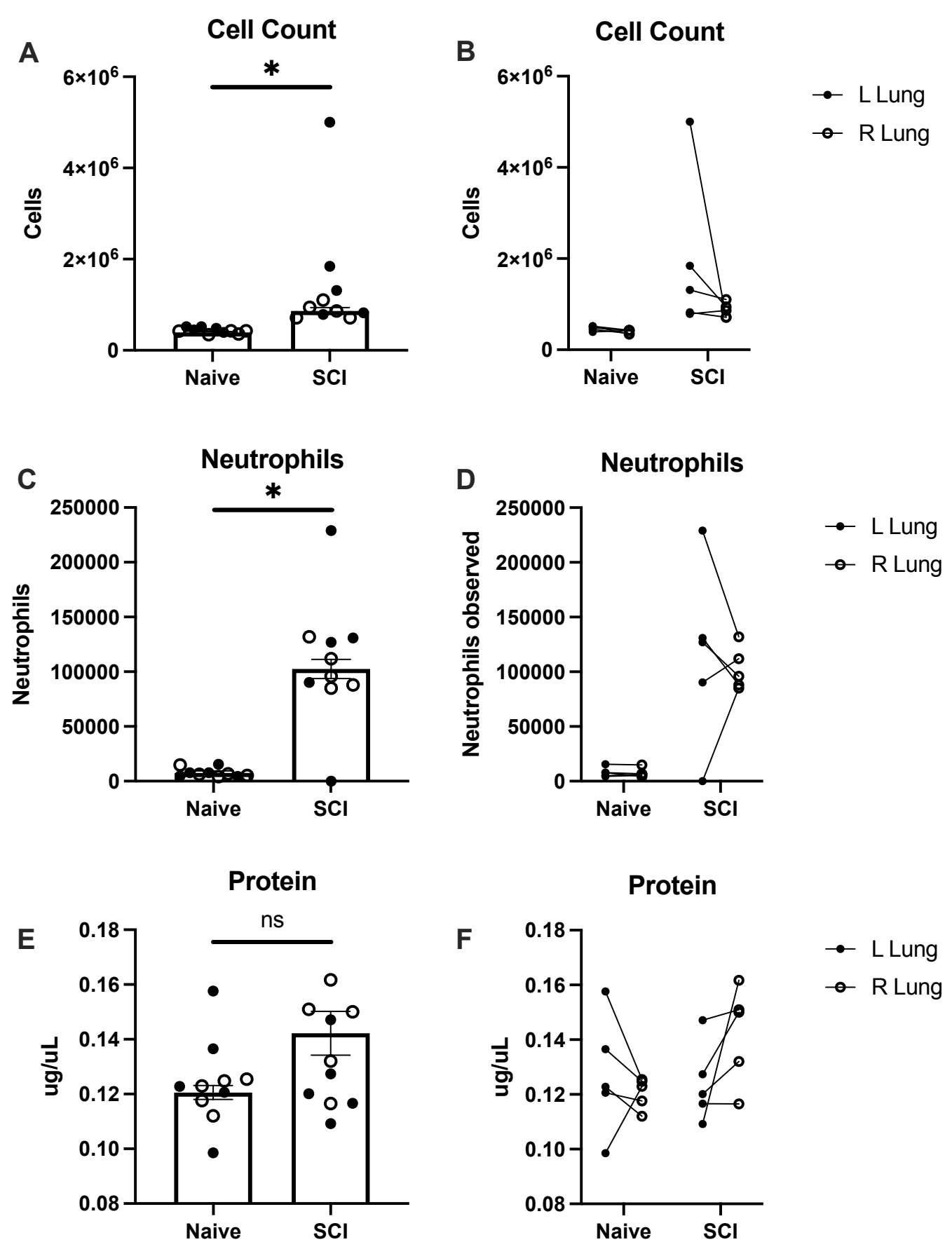

Figure 2.

Changes in cell numbers and neutrophil counts were significantly increased two-weeks post-SCI compared with naïve rats. Measurements of total cell count (A-B), neutrophils (C-D), and protein concentration (E-F) in bronchoalveolar lavage fluid (BAL) from naïve and two weeks post-SCI rats. There was a significant increase in total cell count and neutrophils following SCI (A, C; $\mathrm{N}=10, \mathrm{P}<0.05)$, but no detected change in protein $(\mathrm{E} \mathrm{N}=10, \mathrm{P}>0.05)$. The left lung trends towards a greater cell count and neutrophil levels following SCI (B, D; N=5). However, the significance of this finding was not analyzed due to small Ns. 
A

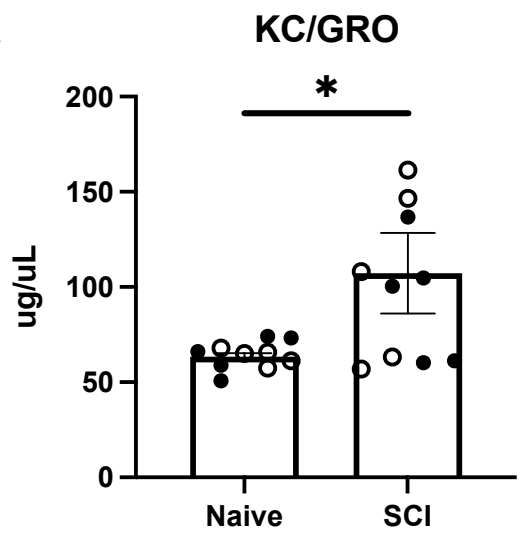

C

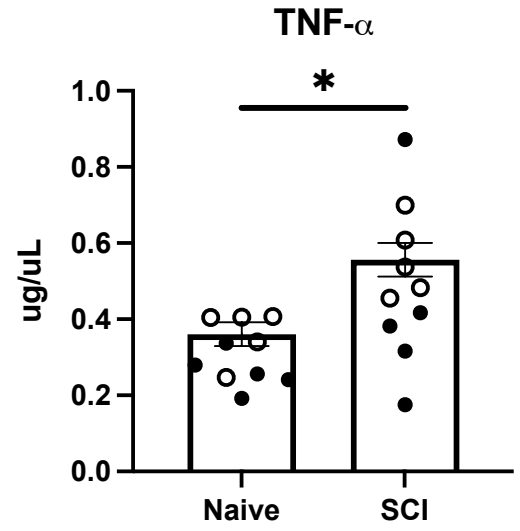

$\mathbf{E}$

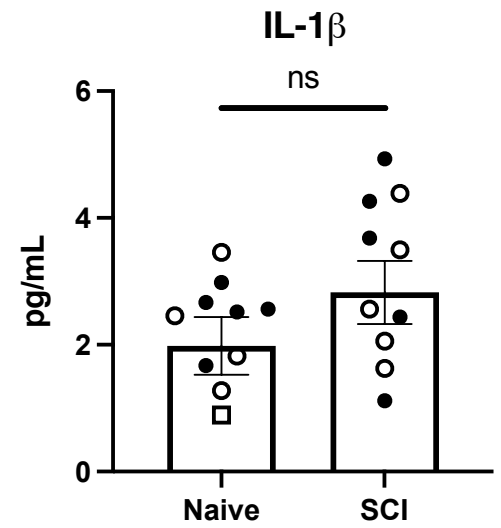

B

KC/GRO

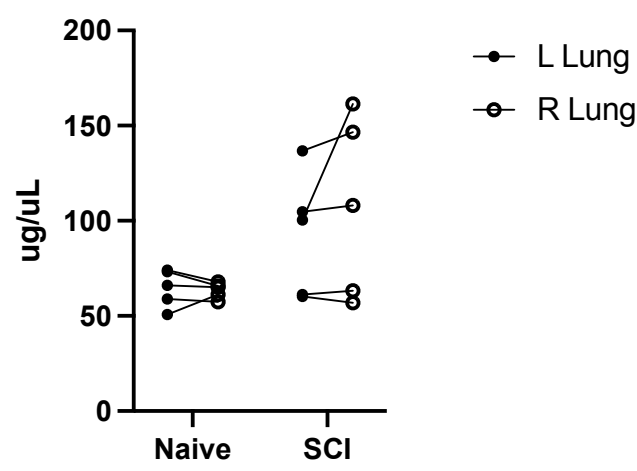

D

TNF- $\alpha$

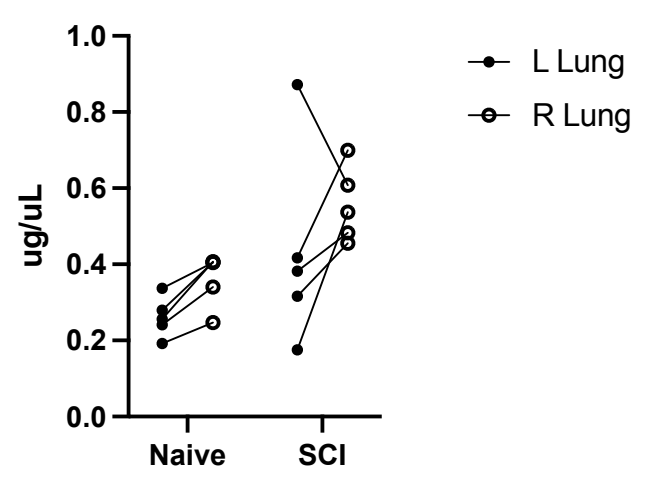

$\mathbf{F}$

IL-1 $\beta$

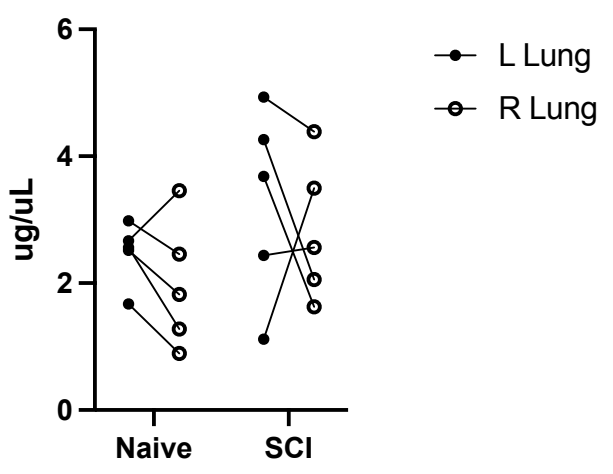

Figure 3.

Levels of KC/GRO and TNF- $\alpha$ were significantly increased two-weeks post-SCI compared with naïve rats. Measurements of KC/GRO (A-B), TNF- $\alpha$ (C-D), and IL-1 $\beta$ (E-F) in bronchoalveolar lavage fluid (BAL) from naïve and two weeks-post SCI rats. There was a significant increase in levels of KC/GRO and TNF- $\alpha$ following SCI (A, C; N=10, P<0.05), however, there was no significant change in levels of IL- $1 \beta$ following SCI. Side-specific differences were not found following SCI due to small Ns (B, D, F; N=5). 


\section{A: Naïve L Lung}

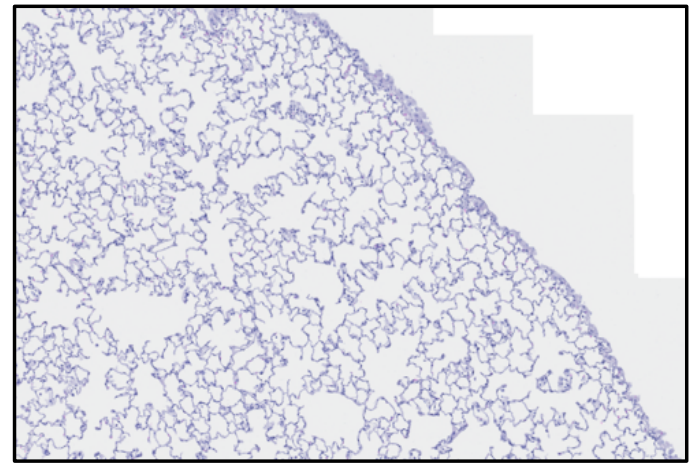

\section{C: Naïve R Lung}

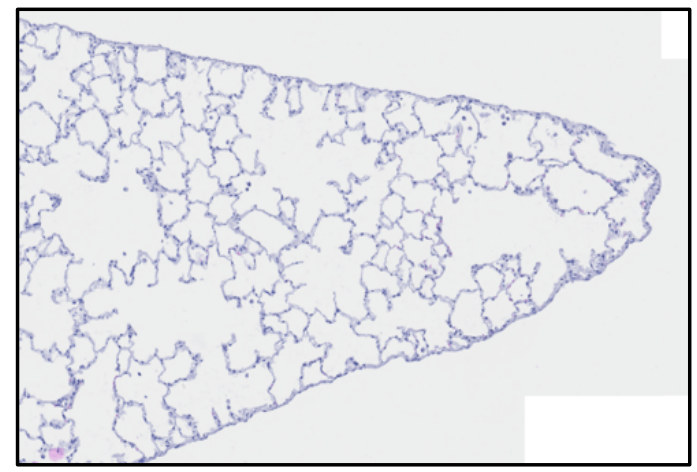

B: SCI L Lung

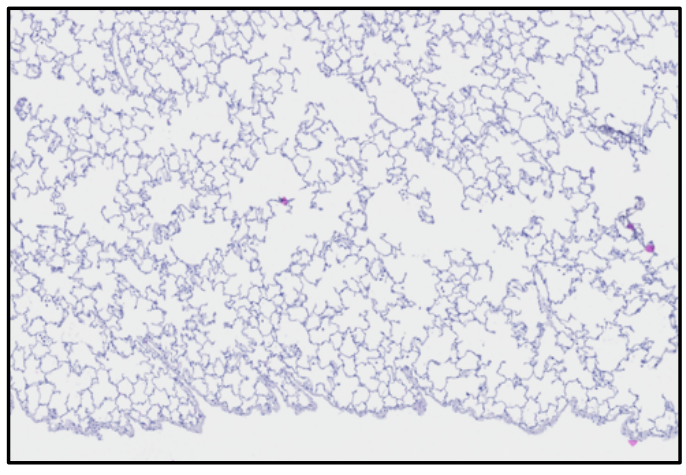

D: SCI R Lung

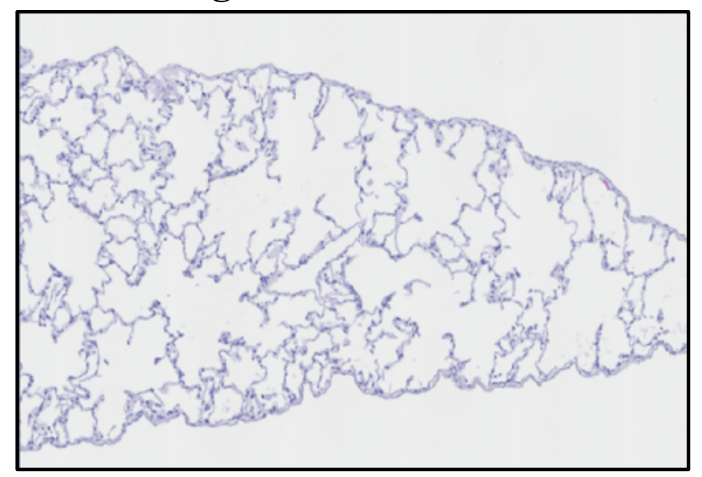

Figure 4.

No histopathologic difference were observed in the lungs from naïve and two weeks post-SCI rats. Lungs were essentially normal (H\&E stain, low power). (A: representative naïve left lung; $\mathrm{N}=5$ ) (B: representative SCI left lung with scattered parabronchial lymphoid aggregates; $\mathrm{N}=5$ ) (C: representative naïve right lung; $\mathrm{N}=5$ ) (D: representative $\mathrm{SCI}$ right lung; $\mathrm{N}=5$ ). 

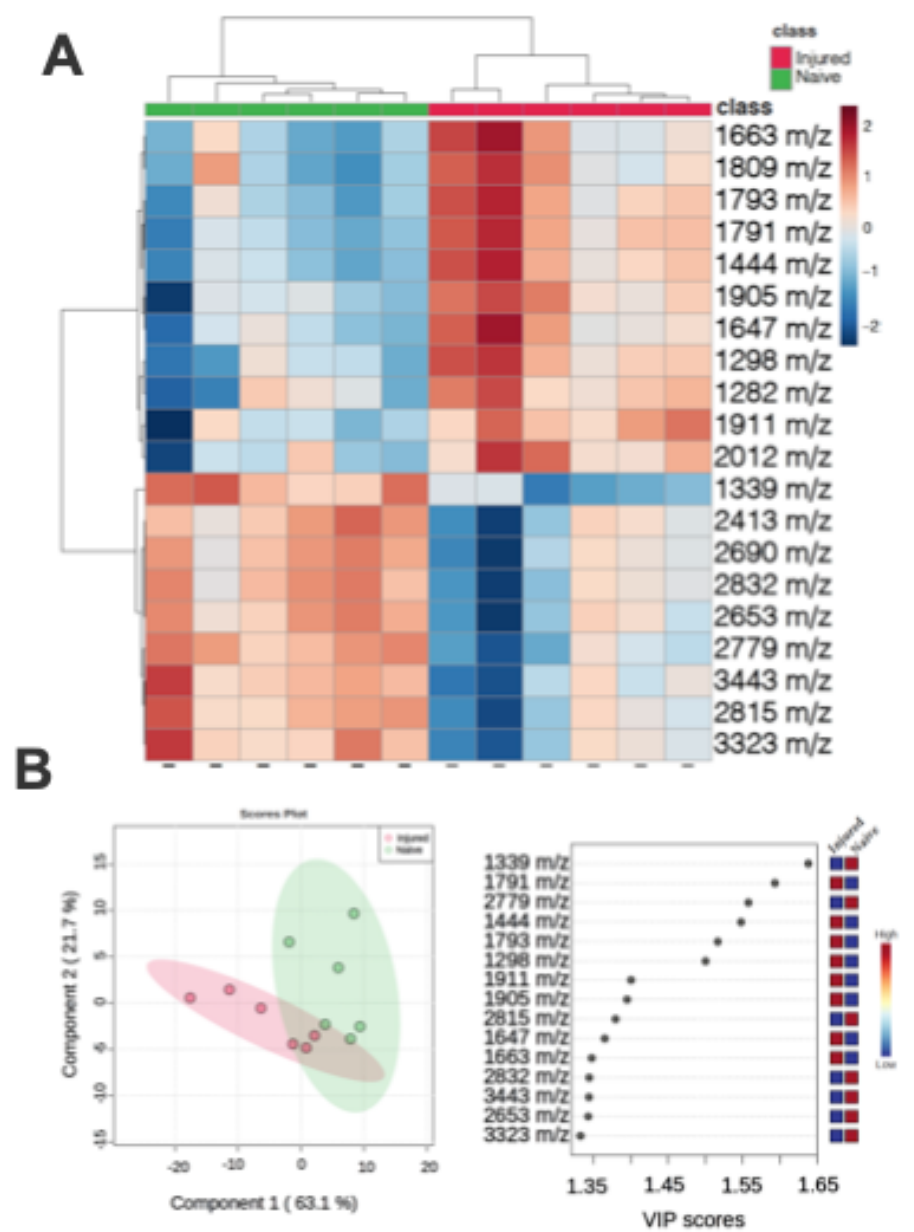

C
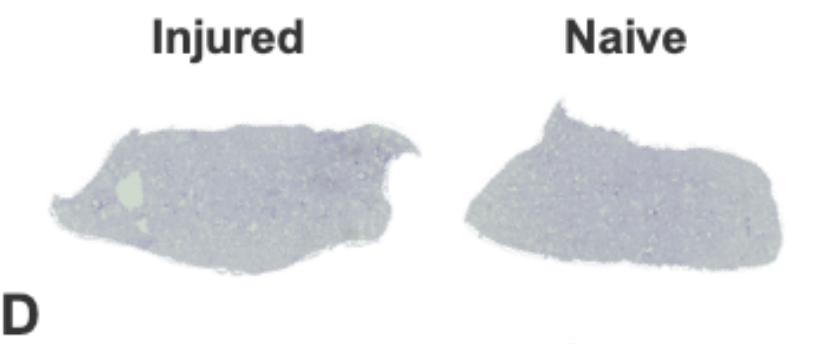

PAS
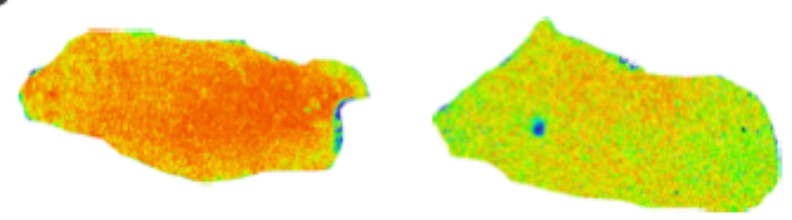

1298
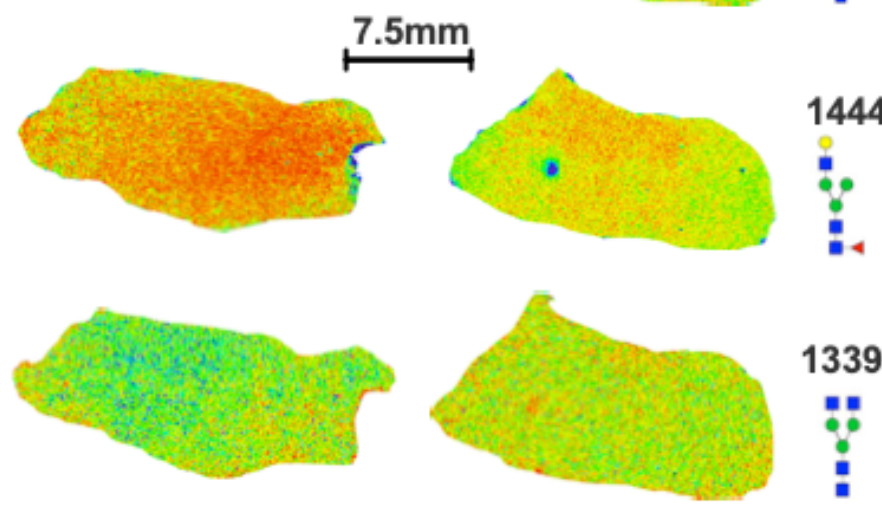

$\min =$

max

Figure 5.

MALDI mass spectrometry imaging analysis of the left lung following SCI ( $=5)$. Multivariant clustering heat analysis shows separation of SCI and naïve lungs using top 25 most changed Nlinked glycans (A). (B left panel) PLS-Da analysis shows 2D clustering of SCI and naïve rat lungs (B left panel). VIP scores of most changed $\mathrm{N}$-glycans in whole lung (B right panel). There was no significant PAS difference the left lungs from $\mathrm{SCI}$ and naïve rats $(\mathrm{C} ; \mathrm{N}=5)$.

Representative images of selected $\mathrm{N}$-glycans and their spatial distribution across the whole lung $(\mathrm{D} ; \mathrm{N}=5)$. 

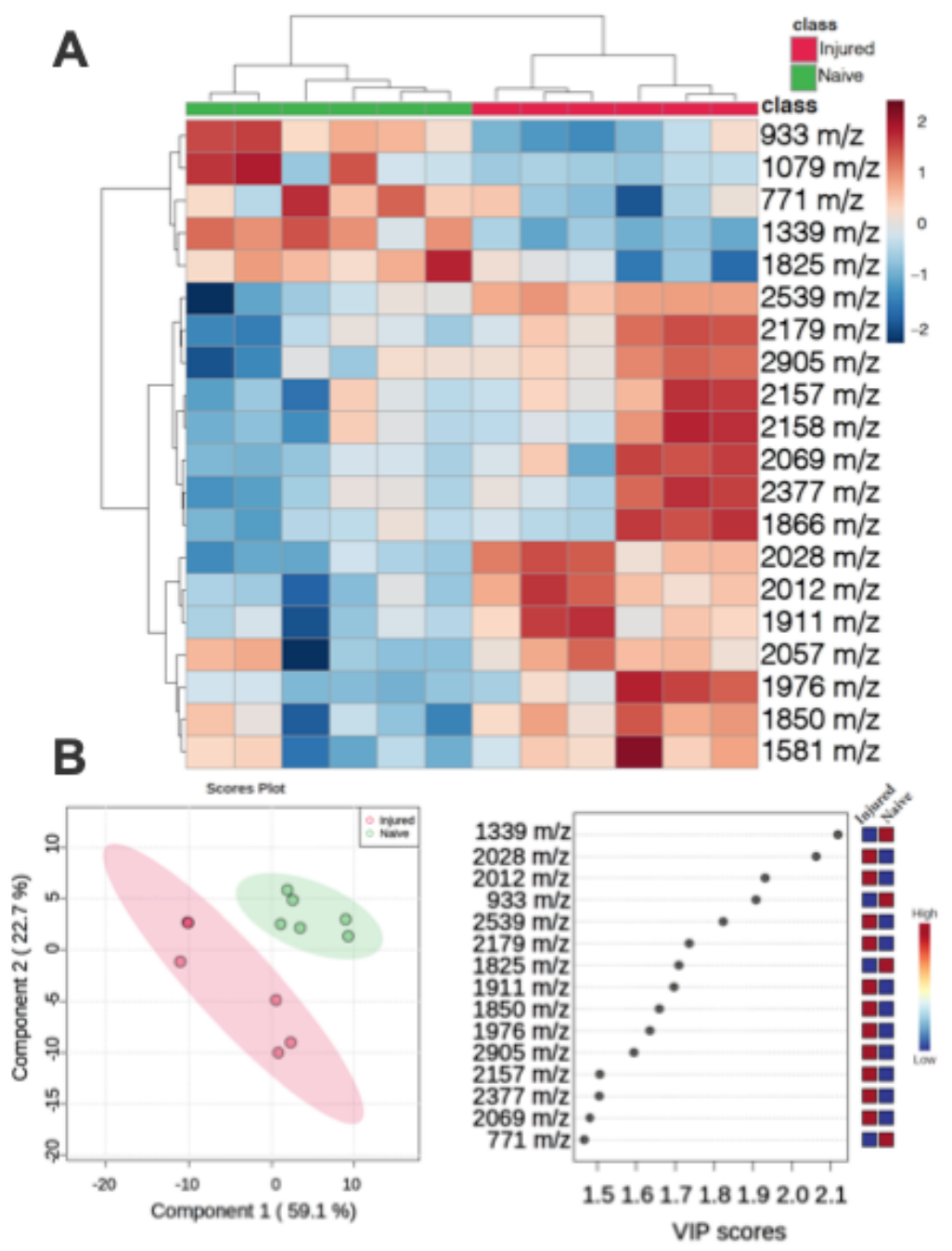

C

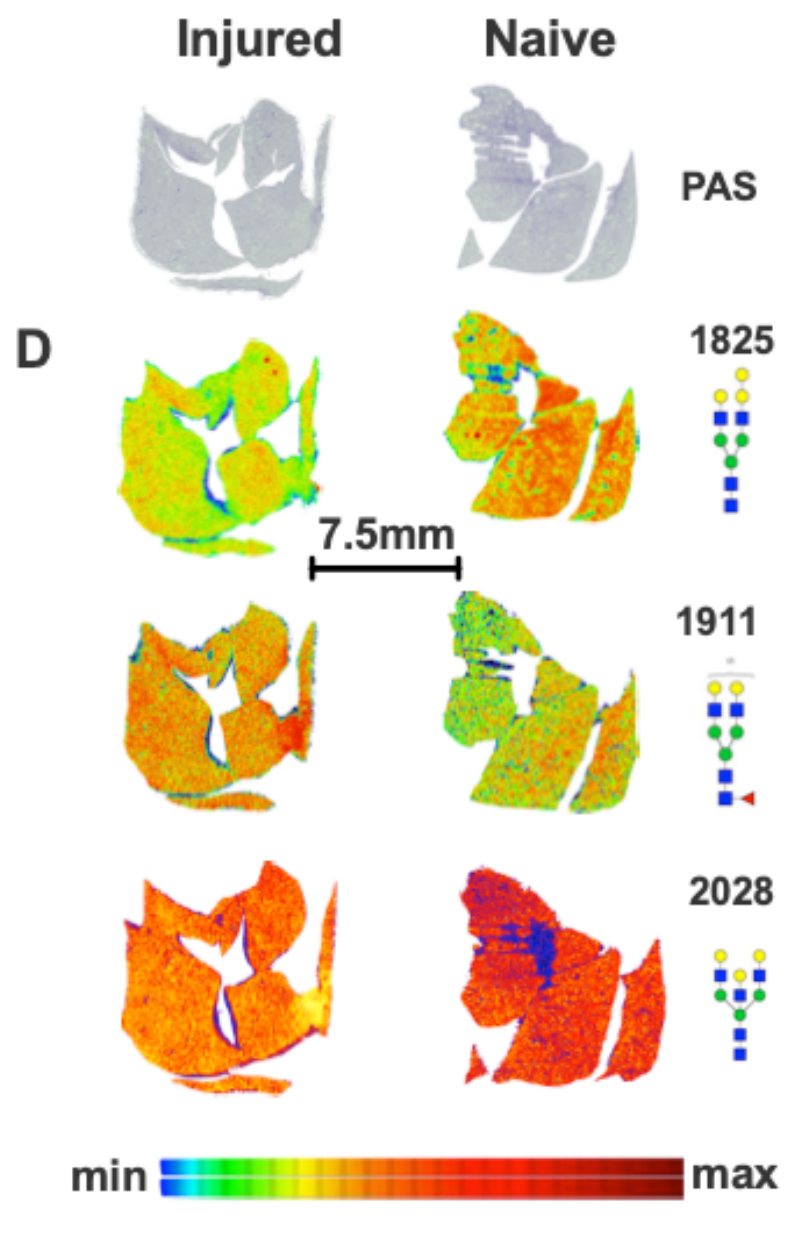

Figure 6.

MALDI mass spectrometry imaging analysis of the right lung following SCI (N=5). Multivariant clustering heat analysis shows separation of SCI and naïve lungs using top 25 most changed Nlinked glycans (A). (B left panel) PLS-Da analysis shows 2D clustering of SCI and naïve rat lungs (B left panel). VIP scores of most changed $\mathrm{N}$-glycans in whole lung (B right panel). There was no significant PAS difference the right lungs from SCI and naïve rats $(C ; N=5)$.

Representative images of selected $\mathrm{N}$-glycans and their spatial distribution across the whole lung $(\mathrm{D} ; \mathrm{N}=5)$. 


\section{References}

1 Brown, R., DiMarco, A. F., Hoit, J. D. \& Garshick, E. Respiratory dysfunction and management in spinal cord injury. Respir Care 51, 853-868; discussion 869-870 (2006).

2 Reines, H. D. \& Harris, R. C. Pulmonary complications of acute spinal cord injuries. Neurosurgery 21, 193-196, doi:10.1227/00006123-198708000-00010 (1987).

3 Yang, X. X., Huang, Z. Q., Li, Z. H., Ren, D. F. \& Tang, J. G. Risk factors and the surgery affection of respiratory complication and its mortality after acute traumatic cervical spinal cord injury. Medicine (Baltimore) 96, e7887, doi:10.1097/md.0000000000007887 (2017).

4 Schilero, G. J., Bauman, W. A. \& Radulovic, M. Traumatic Spinal Cord Injury: Pulmonary Physiologic Principles and Management. Clin Chest Med 39, 411-425, doi:10.1016/j.ccm.2018.02.002 (2018).

5 Schwarz, M. I. \& Albert, R. K. "Imitators" of the ARDS: implications for diagnosis and treatment. Chest 125, 1530-1535, doi:10.1378/chest.125.4.1530 (2004).

6 Santa Cruz, R., Alvarez, L. V., Heredia, R. \& Villarejo, F. Acute Respiratory Distress Syndrome: Mortality in a Single Center According to Different Definitions. J Intensive Care Med 32, 326-332, doi:10.1177/0885066615608159 (2017).

7 Johnson, E. R. \& Matthay, M. A. Acute lung injury: epidemiology, pathogenesis, and treatment. J Aerosol Med Pulm Drug Deliv 23, 243-252, doi:10.1089/jamp.2009.0775 (2010).

8 Rubenfeld, G. D. et al. Incidence and outcomes of acute lung injury. $N$ Engl J Med 353, 1685-1693, doi:10.1056/NEJMoa050333 (2005).

9 Veeravagu, A. et al. Acute respiratory distress syndrome and acute lung injury in patients with vertebral column fracture(s) and spinal cord injury: a nationwide inpatient sample study. Spinal Cord 51, 461-465, doi:10.1038/sc.2013.16 (2013).

10 Veeravagu, A. et al. Acute lung injury in patients with subarachnoid hemorrhage: a nationwide inpatient sample study. World Neurosurg 82, e235-241, doi:10.1016/j.wneu.2014.02.030 (2014).

11 Rincon, F. et al. Impact of acute lung injury and acute respiratory distress syndrome after traumatic brain injury in the United States. Neurosurgery 71, 795-803, doi:10.1227/NEU.0b013e3182672ae5 (2012).

12 Holland, M. C. et al. The development of acute lung injury is associated with worse neurologic outcome in patients with severe traumatic brain injury. J Trauma 55, 106-111, doi:10.1097/01.Ta.0000071620.27375.Be (2003).

13 Gris, D., Hamilton, E. F. \& Weaver, L. C. The systemic inflammatory response after spinal cord injury damages lungs and kidneys. Exp Neurol 211, 259-270, doi:10.1016/j.expneurol.2008.01.033 (2008).

14 Liu, J. et al. Resveratrol attenuates spinal cord injury-induced inflammatory damage in rat lungs. Int J Clin Exp Pathol 8, 1237-1246 (2015).

15 Warren, P. M., Campanaro, C., Jacono, F. J. \& Alilain, W. J. Mid-cervical spinal cord contusion causes robust deficits in respiratory parameters and pattern variability. Exp Neurol 306, 122-131, doi:10.1016/j.expneurol.2018.04.005 (2018).

16 Stanback, A. E. et al. Regional N-glycan and lipid analysis from tissues using MALDImass spectrometry imaging. STAR Protoc 2, 100304, doi:10.1016/j.xpro.2021.100304 (2021). 
17 Chong, J., Wishart, D. S. \& Xia, J. Using MetaboAnalyst 4.0 for Comprehensive and Integrative Metabolomics Data Analysis. Curr Protoc Bioinformatics 68, e86, doi:10.1002/cpbi.86 (2019).

18 Parker, J. C. \& Townsley, M. I. Evaluation of lung injury in rats and mice. Am J Physiol Lung Cell Mol Physiol 286, L231-246, doi:10.1152/ajplung.00049.2003 (2004).

19 Morgan, C. J. Use of proper statistical techniques for research studies with small samples. Am J Physiol Lung Cell Mol Physiol 313, L873-1877, doi:10.1152/ajplung.00238.2017 (2017).

20 Lucas, R., Verin, A. D., Black, S. M. \& Catravas, J. D. Regulators of endothelial and epithelial barrier integrity and function in acute lung injury. Biochem Pharmacol 77, 1763-1772, doi:10.1016/j.bcp.2009.01.014 (2009).

21 Welbourn, R. et al. Role for tumor necrosis factor as mediator of lung injury following lower torso ischemia. J Appl Physiol (1985) 70, 2645-2649, doi:10.1152/jappl.1991.70.6.2645 (1991).

22 Wheeler, A. P., Hardie, W. D. \& Bernard, G. R. The role of cyclooxygenase products in lung injury induced by tumor necrosis factor in sheep. Am Rev Respir Dis 145, 632-639, doi:10.1164/ajrccm/145.3.632 (1992).

23 Ao, X. et al. Radiation produces differential changes in cytokine profiles in radiation lung fibrosis sensitive and resistant mice. J Hematol Oncol 2, 6, doi:10.1186/1756-8722-2-6 (2009).

24 Mc, M. J. Histological demonstration of mucin after periodic acid. Nature 158, 202, doi:10.1038/158202a0 (1946).

25 Hashimoto, K., Gross, B. G. \& Lever, W. F. ANGIOKERATOMA CORPORIS DIFFUSUM (FABRY). HISTOCHEMICAL AND ELECTRON MICROSCOPIC STUDIES OF THE SKIN. J Invest Dermatol 44, 119-128 (1965).

26 Dahr, W., Uhlenbruck, G., Janssen, E. \& Schmalisch, R. Heterogeneity of human cell membrane sialoglycoproteins. Blut 32, 171-184, doi:10.1007/bf00995910 (1976).

27 Grossman, R. G. et al. Incidence and severity of acute complications after spinal cord injury. J Neurosurg Spine 17, 119-128, doi:10.3171/2012.5.Aospine12127 (2012).

28 Jackson, A. B. \& Groomes, T. E. Incidence of respiratory complications following spinal cord injury. Arch Phys Med Rehabil 75, 270-275, doi:10.1016/0003-9993(94)90027-2 (1994).

29 Hsieh, Y. H. et al. Brainstem inflammation modulates the ventilatory pattern and its variability after acute lung injury in rodents. $J$ Physiol 598, 2791-2811, doi:10.1113/jp279177 (2020).

30 Paudel, S. et al. CXCL1 regulates neutrophil homeostasis in pneumonia-derived sepsis caused by Streptococcus pneumoniae serotype 3. Blood 133, 1335-1345, doi:10.1182/blood-2018-10-878082 (2019).

31 Puneet, P., Moochhala, S. \& Bhatia, M. Chemokines in acute respiratory distress syndrome. Am J Physiol Lung Cell Mol Physiol 288, L3-15, doi:10.1152/ajplung.00405.2003 (2005).

32 Kurdowska, A. et al. Anti-interleukin 8 autoantibody: interleukin 8 complexes in the acute respiratory distress syndrome. Relationship between the complexes and clinical disease activity. Am J Respir Crit Care Med 163, 463-468, doi:10.1164/ajrccm.163.2.2005109 (2001). 
33 Son, D. S., Parl, A. K., Rice, V. M. \& Khabele, D. Keratinocyte chemoattractant $(\mathrm{KC})$ /human growth-regulated oncogene (GRO) chemokines and pro-inflammatory chemokine networks in mouse and human ovarian epithelial cancer cells. Cancer Biol Ther 6, 1302-1312, doi:10.4161/cbt.6.8.4506 (2007).

34 Matuschak, G. M. \& Lechner, A. J. Acute lung injury and the acute respiratory distress syndrome: pathophysiology and treatment. Mo Med 107, 252-258 (2010).

35 Crosby, L. M. \& Waters, C. M. Epithelial repair mechanisms in the lung. Am J Physiol Lung Cell Mol Physiol 298, L715-731, doi:10.1152/ajplung.00361.2009 (2010).

36 Blank, R. \& Napolitano, L. M. Epidemiology of ARDS and ALI. Crit Care Clin 27, 439458, doi:10.1016/j.ccc.2011.05.005 (2011).

37 Ioffe, E., Liu, Y. \& Stanley, P. Essential role for complex N-glycans in forming an organized layer of bronchial epithelium. Proc Natl Acad Sci U S A 93, 11041-11046, doi:10.1073/pnas.93.20.11041 (1996).

38 Dennis, J. W., Granovsky, M. \& Warren, C. E. Protein glycosylation in development and disease. Bioessays 21, 412-421, doi:10.1002/(sici)1521-1878(199905)21:5<412::Aidbies8>3.0.Co;2-5 (1999).

39 Carter, C. L. et al. MALDI-MSI spatially maps N-glycan alterations to histologically distinct pulmonary pathologies following irradiation. Sci Rep 10, 11559, doi:10.1038/s41598-020-68508-y (2020).

40 Lamblin, G. et al. Human airway mucin glycosylation: a combinatory of carbohydrate determinants which vary in cystic fibrosis. Glycoconj $J$ 18, 661-684, doi:10.1023/a:1020867221861 (2001).

41 Whitsett, J. A. et al. Glycosylation and secretion of surfactant-associated glycoprotein A. J Biol Chem 260, 15273-15279 (1985).

42 Matute-Bello, G., Frevert, C. W. \& Martin, T. R. Animal models of acute lung injury. Am J Physiol Lung Cell Mol Physiol 295, L379-399, doi:10.1152/ajplung.00010.2008 (2008).

43 Perl, M. et al. Fas-induced pulmonary apoptosis and inflammation during indirect acute lung injury. Am J Respir Crit Care Med 176, 591-601, doi:10.1164/rccm.2006111743OC (2007).

44 Bao, F., Brown, A., Dekaban, G. A., Omana, V. \& Weaver, L. C. CD11d integrin blockade reduces the systemic inflammatory response syndrome after spinal cord injury. Exp Neurol 231, 272-283, doi:10.1016/j.expneurol.2011.07.001 (2011).

45 Sun, X. et al. Multiple organ dysfunction and systemic inflammation after spinal cord injury: a complex relationship. J Neuroinflammation 13, 260, doi:10.1186/s12974-0160736-y (2016).

46 He, B. \& Nan, G. Pulmonary edema and hemorrhage after acute spinal cord injury in rats. Spine J 16, 547-551, doi:10.1016/j.spinee.2015.11.065 (2016).

47 Lerch, J. K., Puga, D. A., Bloom, O. \& Popovich, P. G. Glucocorticoids and macrophage migration inhibitory factor (MIF) are neuroendocrine modulators of inflammation and neuropathic pain after spinal cord injury. Semin Immunol 26, 409-414, doi:10.1016/j.smim.2014.03.004 (2014).

48 Bernard, G. R. et al. The American-European Consensus Conference on ARDS. Definitions, mechanisms, relevant outcomes, and clinical trial coordination. Am J Respir Crit Care Med 149, 818-824, doi:10.1164/ajrccm.149.3.7509706 (1994). 
49 Ho, W. L., Hsu, W. M., Huang, M. C., Kadomatsu, K. \& Nakagawara, A. Protein glycosylation in cancers and its potential therapeutic applications in neuroblastoma. $J$ Hematol Oncol 9, 100, doi:10.1186/s13045-016-0334-6 (2016). 